\title{
Changes in cancer incidence and mortality in Australia over the period 1996-2015
}

\author{
Qingwei Luo ${ }^{1,2^{*}} \mathbb{D}$, Julia Steinberg ${ }^{1,2}$, Dianne L. O'Connell ${ }^{1,2,3}$, Paul B. Grogan ${ }^{1,2}$, Karen Canfell ${ }^{1,2,4}$ \\ and Eleonora Feletto ${ }^{1,2}$
}

\begin{abstract}
Objective: A previous Australian study compared the observed numbers of cancer cases and deaths in 2007 with the expected numbers based on 1987 rates. This study examines the impact of cancer rate changes over the 20-year period 1996-2015, for people aged under 75 years.

Results: The overall age-standardised cancer incidence rate increased from 350.7 in 1995 to 364.4 per 100,000 in 2015. Over the period 1996-2015, there were 29,226 (2.0\%) more cases (males: 5940, 0.7\%; females: 23,286, 3.7\%) than expected numbers based on 1995 rates. Smaller numbers of cases were observed compared to those expected for cancers of the lung for males and colorectum, and cancers with unknown primary. Larger numbers of cases were observed compared to those expected for cancers of the prostate, thyroid and female breast. The overall age-standardised cancer mortality rate decreased from 125.6 in 1995 to 84.3 per 100,000 in 2015. During 1996 to 2015 there were 106,903 (-20.6\%) fewer cancer deaths (males: - 69,007, - 22.6\%; females: $-37,896,-17.9 \%$ ) than expected based on the 1995 mortality rates. Smaller numbers of deaths were observed compared to those expected for cancers of the lung, colorectum and female breast, and more cancer deaths were observed for liver cancer.
\end{abstract}

Keywords: Australia, Cancer incidence, Cancer mortality, Cancer control

\section{Introduction}

Australia has one of the highest cancer incidence rates in the world [1]. A previous Australian study compared numbers of cancer cases and deaths observed in 2007 with expected numbers based on 1987 rates, with much of the difference likely to be due to the impact of cancer control measures [2]. This prior study showed that over the period 1987-2007, for Australians aged under 75, the number of cancer deaths was 61,000 lower than it would have been based on the 1987 rates [2]. The number of cancer cases, however, was greater than would have been expected based on 1987 rates [2]. It was posited that improvements in prevention, early detection and treatments played an integral role in the mortality reduction.

\footnotetext{
*Correspondence: qingweil@nswcc.org.au

${ }^{1}$ Cancer Research Division, Cancer Council NSW, Kings Cross, PO Box 572, Sydney, NSW 1340, Australia

Full list of author information is available at the end of the article
}

Some of the increased incidence was associated with increased use of medical services, such as the widespread uptake from the early 1990s of prostate specific antigen (PSA) testing to assess prostate cancer risk [2].

There have been significant developments in cancer control in Australia over the past few decades [2]. Tobacco control was one of the most successful, which through reduced prevalence of smoking is estimated to have prevented 78,000 deaths from lung cancer alone between 1956 and 2015 [3, 4]. Additionally, organised early detection is provided through national screening programs for cancers of the cervix, breast and colorectum, which have all experienced reductions in mortality [5]. The continued development of cancer control initiatives based on the most recent evidence is vital. To identify areas that warrant further attention this analysis provides more recent summary measures of changes 
from 1996 to 2015 in cancer incidence and mortality for Australian people aged under 75 years.

\section{Main text Methods}

Population and tabulated data with the numbers of new cancer cases and deaths by sex, 5-year age group and calendar year from 1994 to 2016 for all cancers combined (ICD-10 codes C00-C97, D45, D46, D47.1, D47.3-D47.5) and for 23 individual cancer types were obtained from the Australian Institute of Health and Welfare (AIHW) $[6,7]$. The remaining cancer types were grouped as 'other'. All cancers for children aged 0-14 years were grouped to avoid small numbers. The analyses were restricted to those aged less than 75 years because by default cancer is a more prevalent cause of death for older people who have survived premature mortality from other chronic diseases, data on cause of death and diagnoses are less reliable at older ages, screening programs stop at approximately 74 years of age, and treatment regimens tend to be less aggressive for older patients [2]. Data were smoothed using a three-year moving average to reduce random variation by single year, and 1995 was used as the reference year.

\section{Statistical analysis}

The methods have been described previously [2]. In brief, Joinpoint regression analysis was used to test for significant changes in cancer rates and estimate the average annual percentage change (AAPC) over the period from 1995 to 2015 [8]. We also reported the annual percentage change (APC) over the last period identified by Joinpoint. We estimated the change in numbers of cancer cases and deaths due to changes in cancer rates rather than changes in the population size and ageing. To do this, we first estimated the expected numbers of cancer cases and deaths for the 20-year period 1996-2015 if rates had remained at 1995 levels, by multiplying the sex-age-specific rates in 1995 by the estimated populations in 1996-2015 [2]. We then calculated the differences in the expected cumulative numbers of cases and deaths and the corresponding observed numbers [2]. All rates were age-standardised to the Australian 2001 population. Statistical analyses were performed using Joinpoint 4.7.0.0 [8] and STATA [9].

\section{Results}

\section{Cancer incidence}

The overall age-standardised incidence rate for all cancers combined increased by $3.9 \%$ from 350.7 in 1995 to 364.4 per 100,000 in 2015 (Fig. 1a and Table 1A). Different patterns were observed for males and females, with the incidence rate for all cancers combined for males showing fluctuations over the 20-year period (AAPC: $-0.1,95 \% \mathrm{CI}-0.4,0.2)$, while a significant increase in the incidence rate for all cancers combined was observed for females (AAPC: 0.5, 95\% CI 0.4, 0.5). Notably, in the last period identified by Joinpoint there were opposing changes in cancer incidence rates for males and females, with a decrease observed for males (APC: -1.4 , 95\% CI $-1.6,-1.3$ in 2008-2015) and an increase observed for females (APC: 0.9, 95\% CI 0.7, 1.0 in 2009-2015) (Table 1A). During 1996-2015, a total of 29,226 (2.0\%) more cases (males: 5,940, 0.7\%; females: 23,286, 3.7\%) were observed compared to the expected numbers based on 1995 rates (Table 2A). For males, compared with expected, the largest differences with smaller numbers of cases observed were for lung $(-23,156,-23.8 \%)$ and colorectal $(-9,092-8.5 \%)$ cancer, while larger numbers of cases were observed for prostate cancer $(29,638$, $14.8 \%)$ and melanoma $(7,208,8.6 \%)$. For females, compared with expected, smaller numbers of cases were observed for cervical cancer $(-7,405,-34.7 \%)$ and cancers with unknown primary (CUP) $(-6,123,-35.8 \%)$, while larger numbers of cases were observed for thyroid $(10,993,83.6 \% \%)$ and breast $(9,952,4.9 \%)$ cancers.

\section{Cancer mortality}

The age-standardised mortality rate for all cancers combined decreased by $32.9 \%$ from 125.6 in 1995 to 84.3 per 100,000 in 2015 (Fig. 1b and Table 1B). Significant decreases in mortality rates were observed for both males (AAPC: $-2.2,95 \% \mathrm{CI}-2.3,-2.2$ ) and females (AAPC: $-1.7,95 \%$ CI $-1.8,-1.7)$. Mortality rates for most cancer types, except liver cancer, decreased throughout the 20 -year period. There were only negligible changes in mortality rates for cancers of the pancreas, thyroid, corpus uteri and uterus over the whole study period 19952015. However, a significant increase in mortality rates for cancer of corpus uteri and uterus in the last period from 2010 to 2015 was identified by Joinpoint (Table 1B). During 1996-2015 there were 106,903 fewer cancer

(See figure on next page.)

Fig. 1 Trends in age-standardised incidence and mortality rates in Australia for 1995-2015 from Joinpoint regression analysis for people aged under 75 years (a Incidence; $\mathbf{b}$ mortality). All rates were age-standardised to the Australian 2001 population. $\mathrm{p}<0.05$ was considered to indicate statistical significance for the Joinpoint analysis of changes in rates. C26.0 ("malignant neoplasms of the intestinal tract unspecified") was included for colorectal cancer mortality, as it has been reported that deaths certified as 'bowel cancer'tend to be coded as C26.0 [7] 


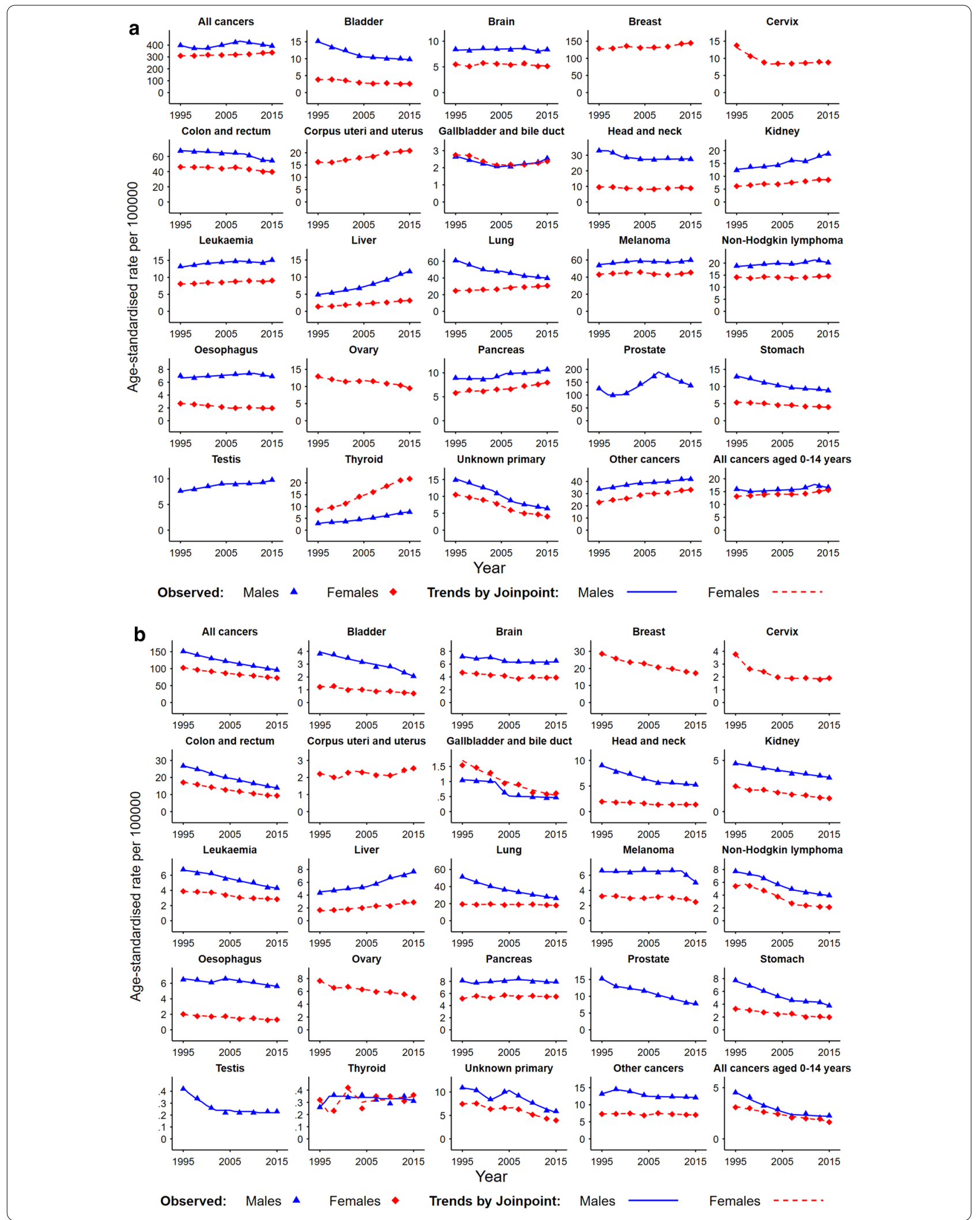




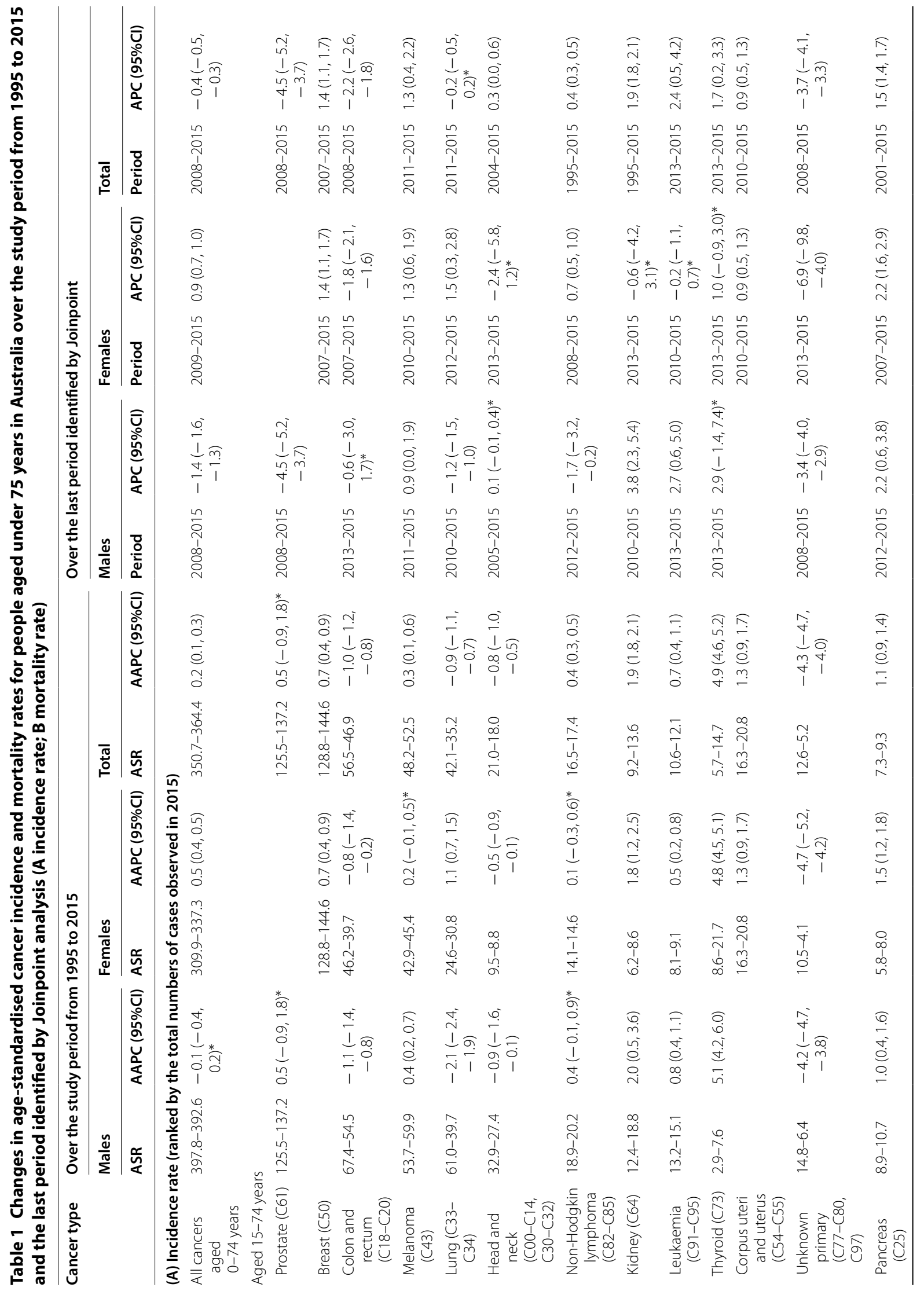


\begin{tabular}{l|llllllll} 
& 0 & 0 & 0
\end{tabular}

命

व.

곡ㄱ

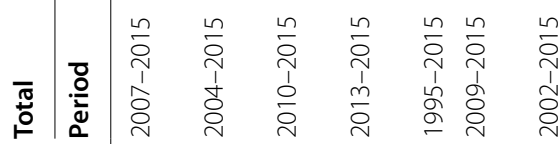

는 $\frac{n}{\circ}$

ㄴ.

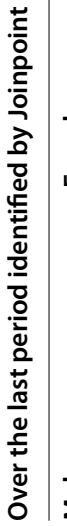
บิ ที

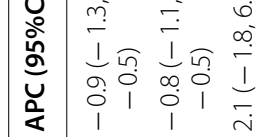

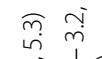

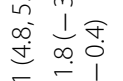

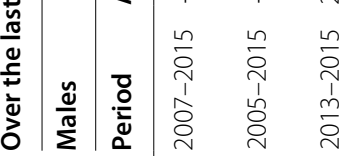

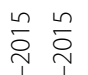

啇 $\frac{1}{\bar{\alpha}}$

는

$\stackrel{1}{\stackrel{1}{c}} \stackrel{m}{\frac{m}{\sigma}}$

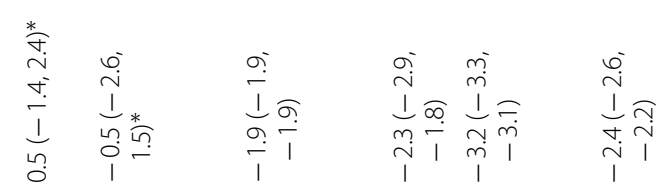

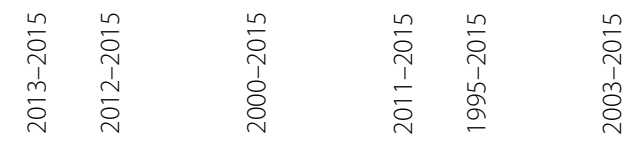

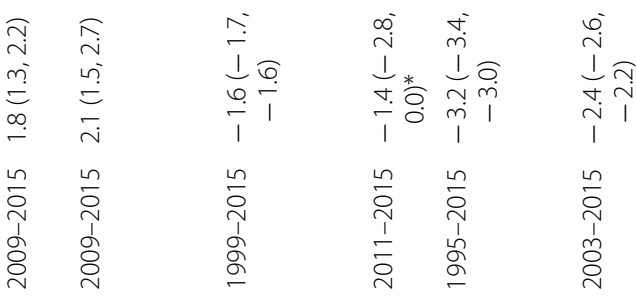

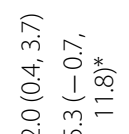

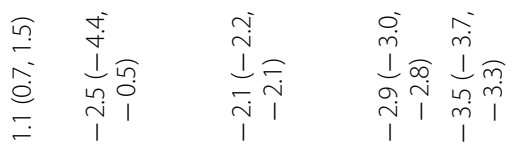

$\stackrel{n}{\stackrel{n}{2}} \stackrel{n}{\circ}$

ำ

in $\stackrel{n}{i} \stackrel{n}{\frac{n}{2}}$

芩

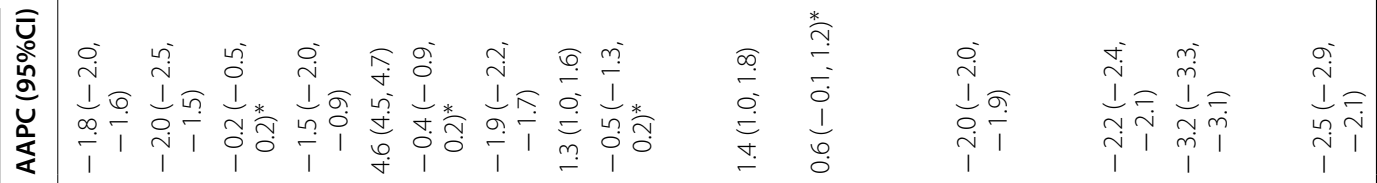

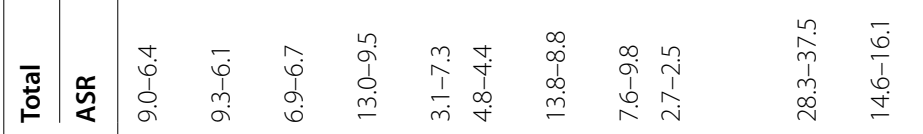

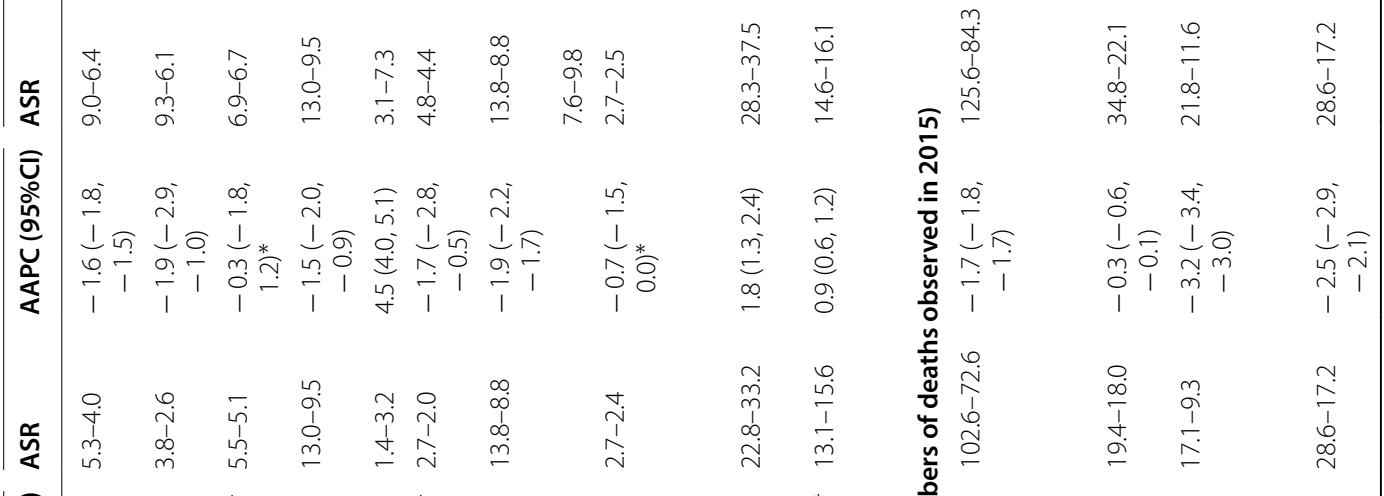

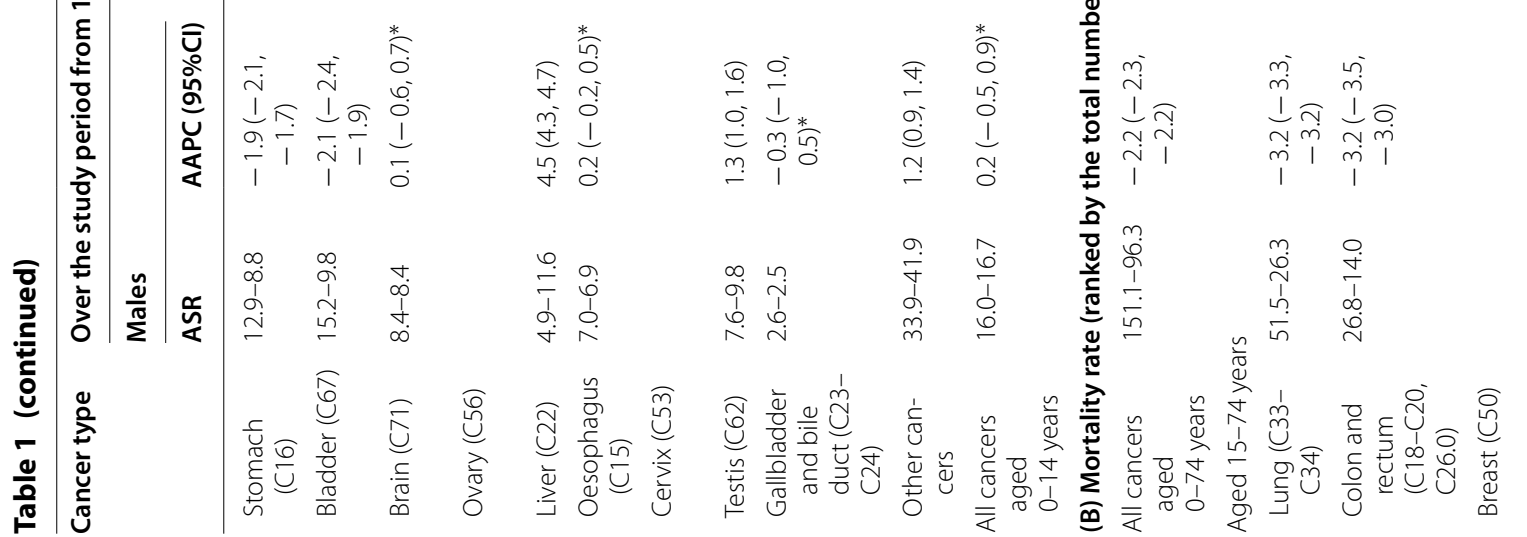




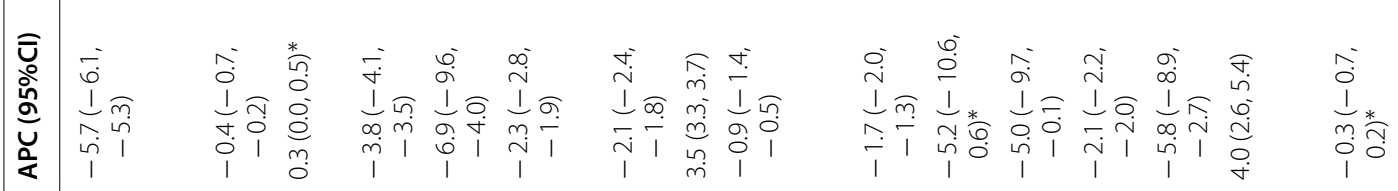

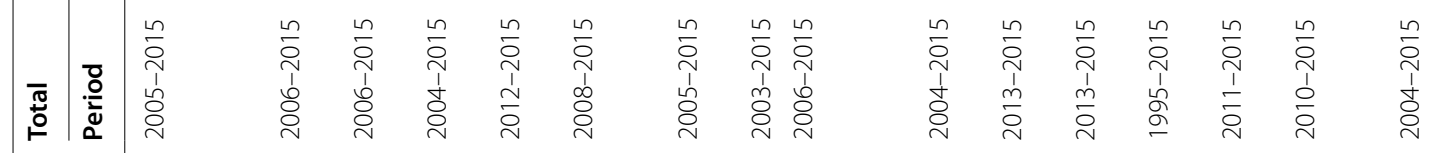

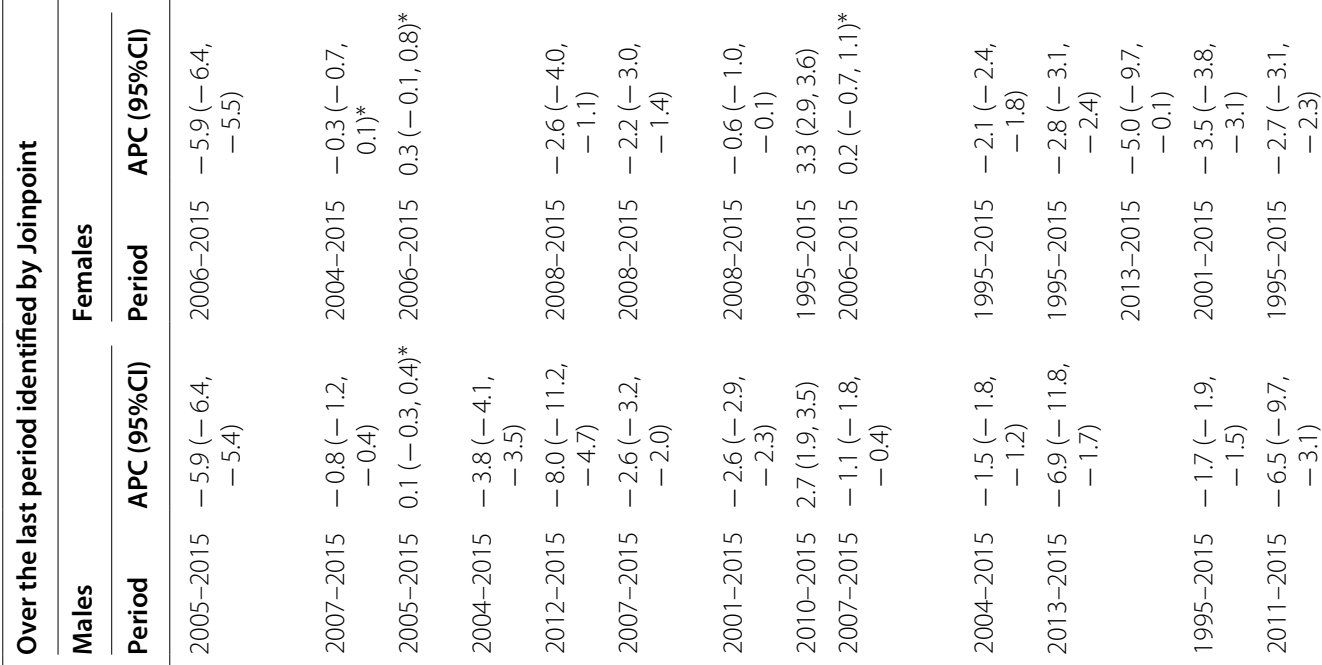

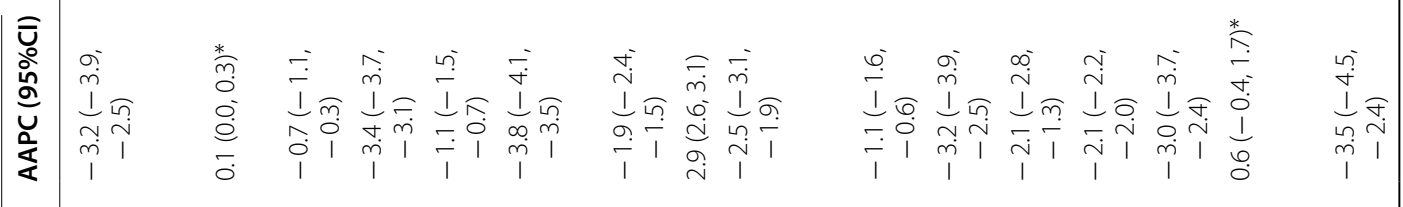

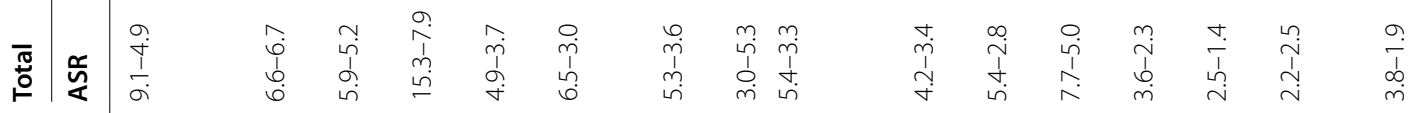

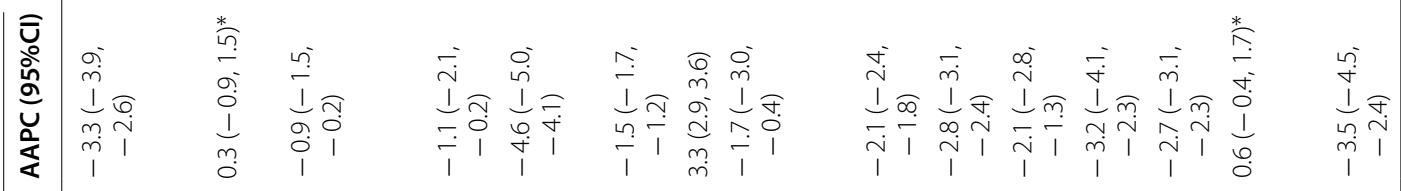

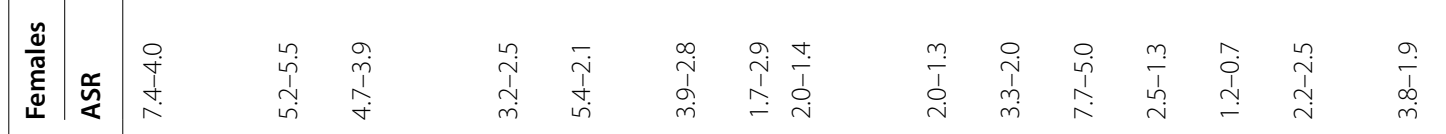

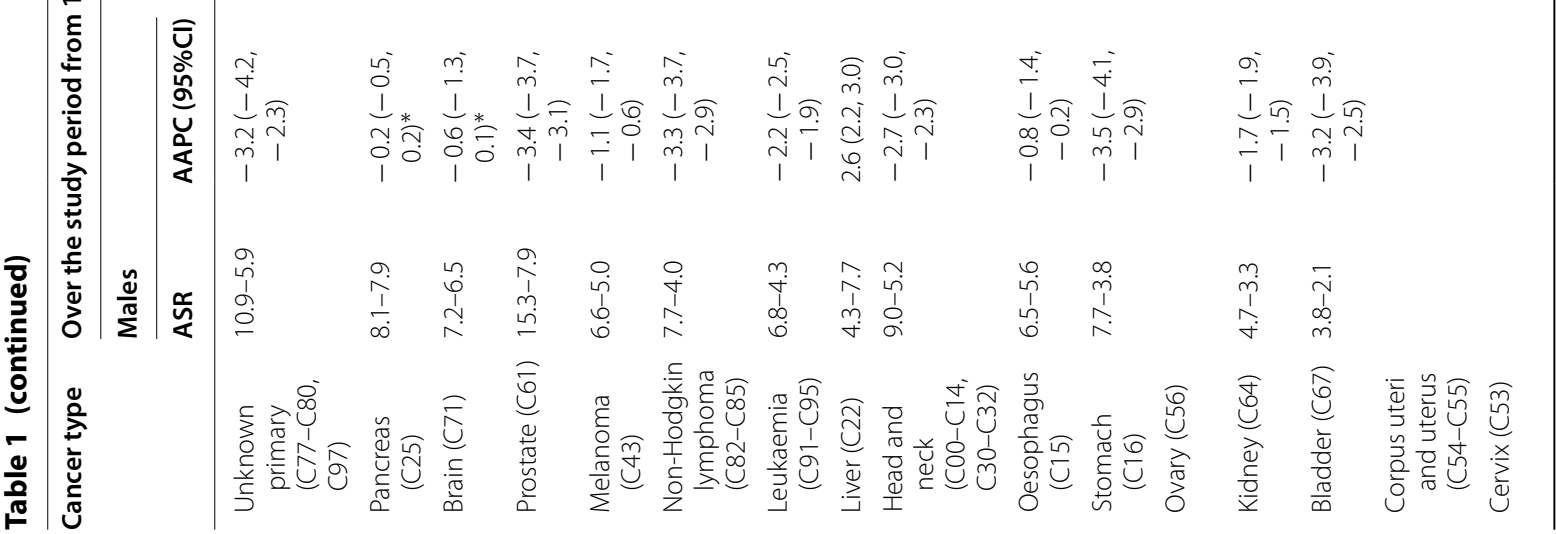




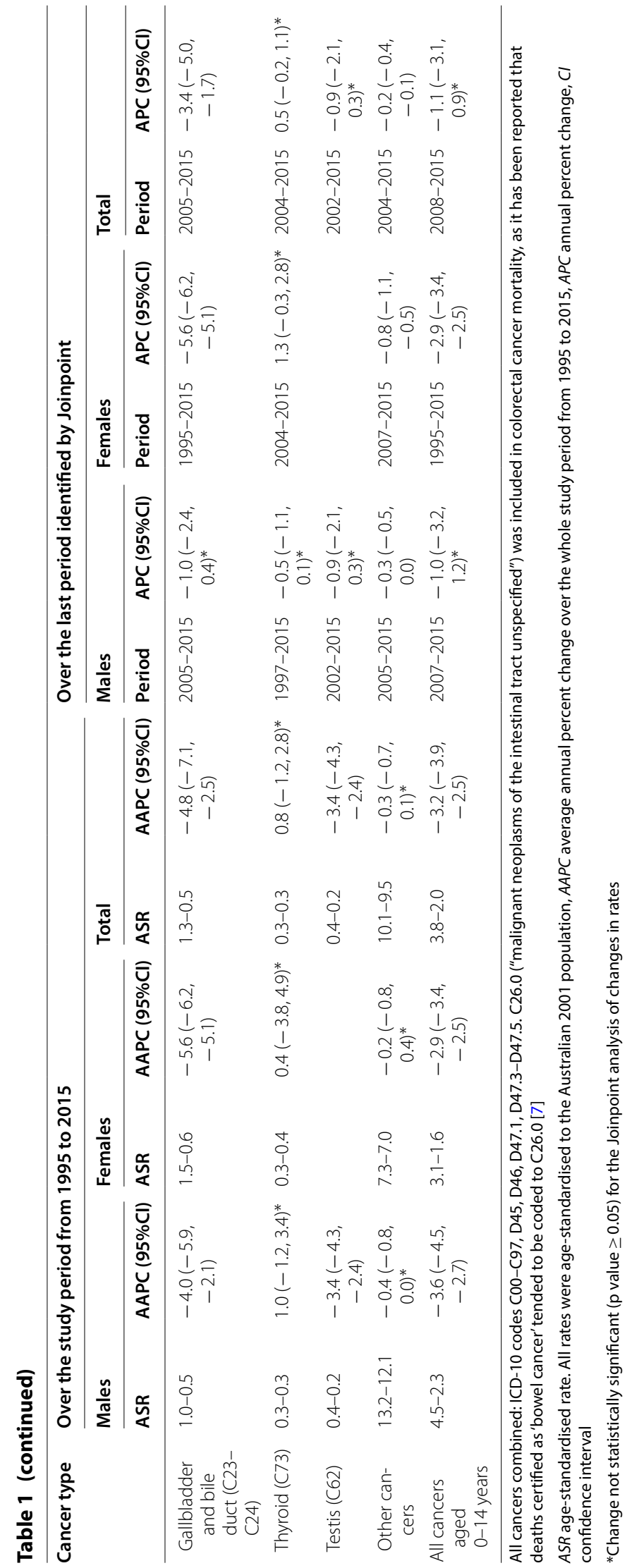




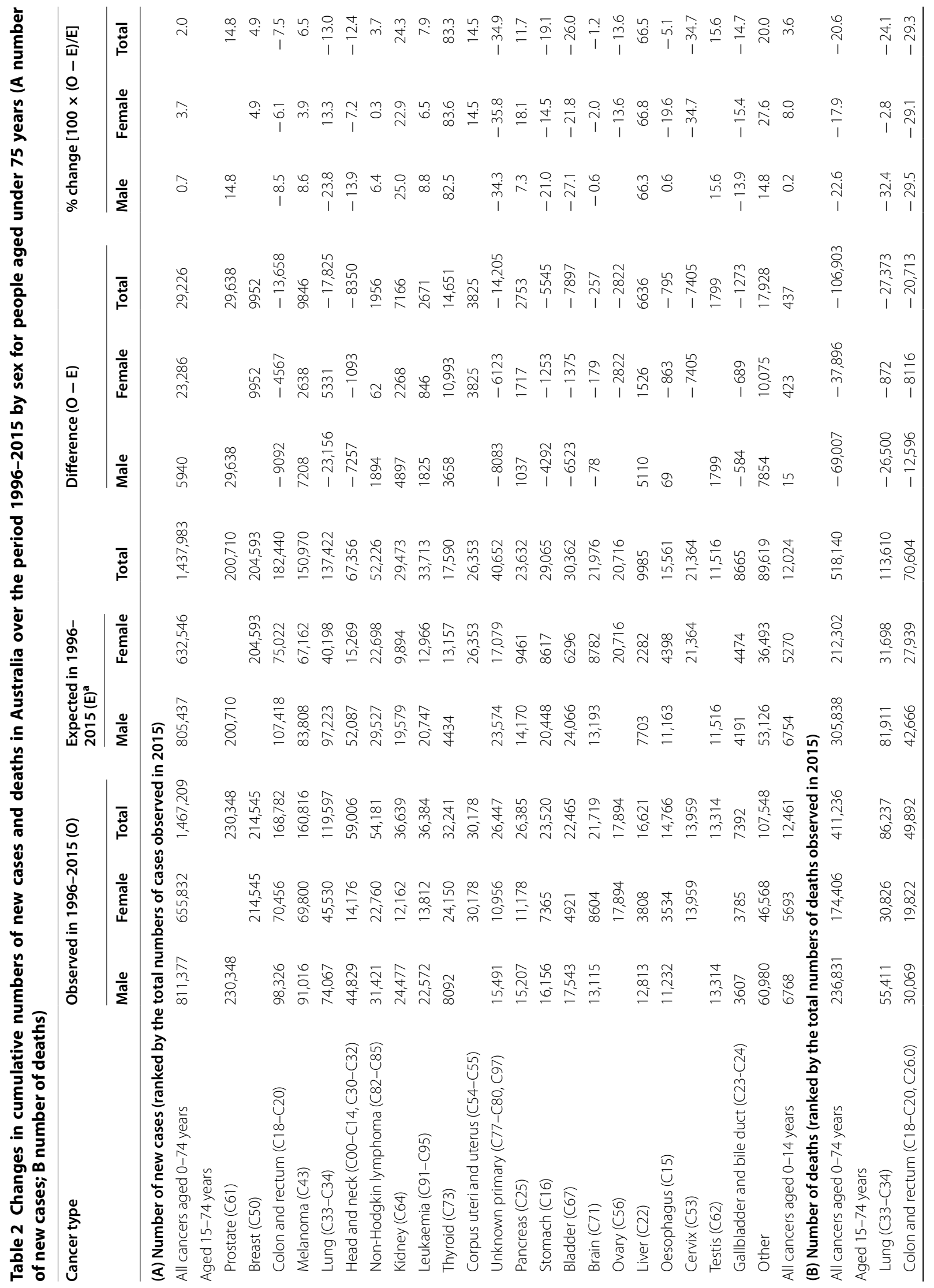




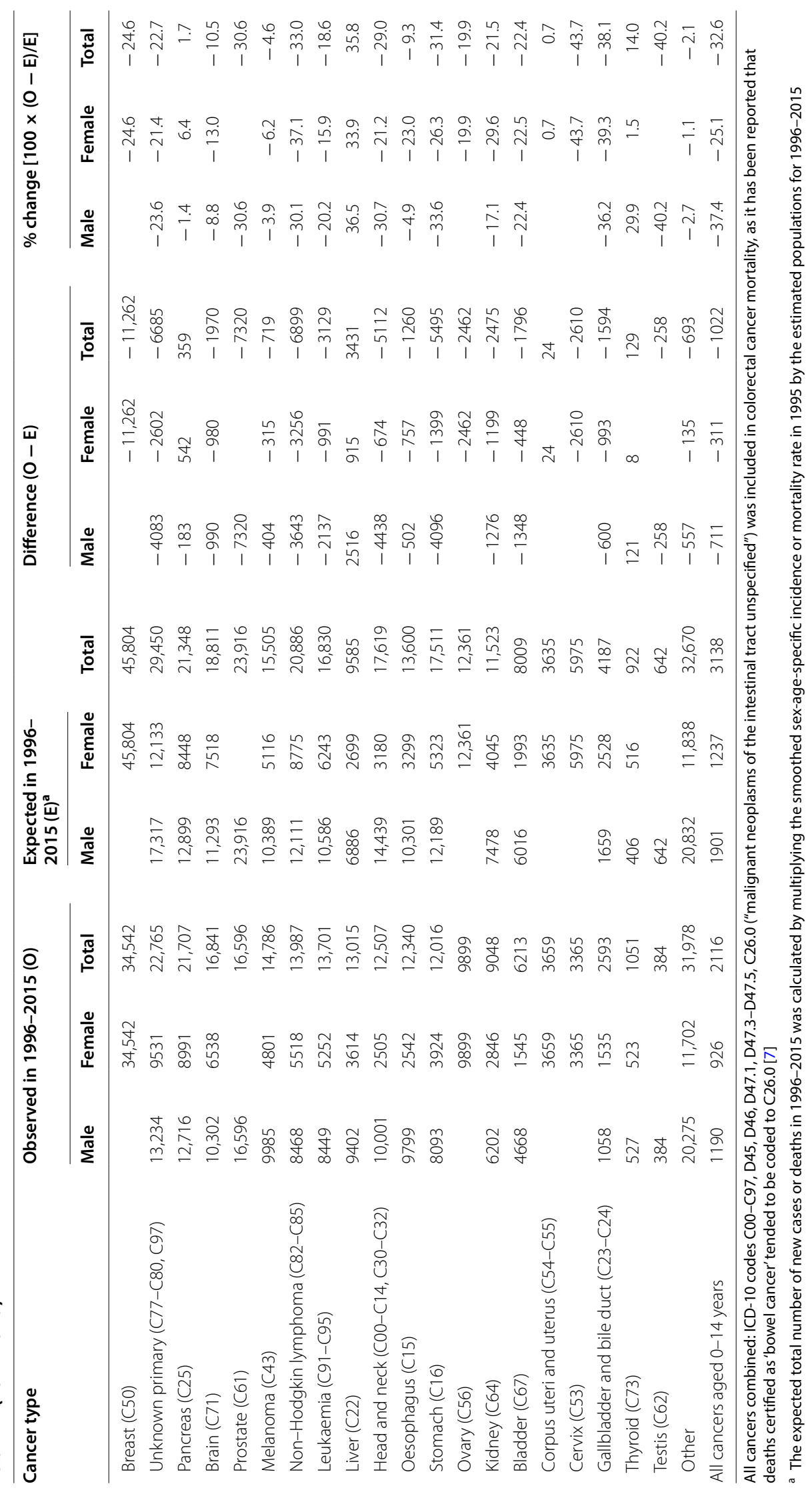


deaths (males: $-69,007,-22.6 \%$; females: $-37,896$, $-17.9 \%)$ than expected based on the 1995 mortality rates (Table 2B). For males, compared with expected, the largest differences were for lung $(-26,500,-32.4 \%)$ and colorectal $(-12,596,-29.5 \%)$ cancers. For females compared with expected, the largest differences were for breast $(-11,262,-24.6 \%)$ and colorectal $(-8,116$, $-29.1 \%$ ) cancers. Liver cancer was the only cancer with a significantly larger number of deaths compared to that expected (3.431, 35.8\%) (Table 2B).

\section{Discussion}

Our results indicated that the overall incidence rate for all cancers for Australians aged under 75 years was relatively stable from 1995 to 2015, while the mortality rate for all cancers declined by $32.9 \%$ over the same period. Over the period 1996-2015, the number of cancer cases was $2 \%$ higher and the number of cancer deaths was 20.6\% lower compared to those expected based on 1995 rates. The patterns observed in the current study were somewhat consistent with those reported for 1987-2007 [2]. However, there was a greater decrease in the cancer mortality rate and a much smaller increase in the cancer incidence rate [2]. These differences are likely to be attributable to multiple factors including risk factor control, changes in diagnostic practices and cancer screening activities, as well as improvements in cancer treatments, which were highlighted in the previous study [2]. Broadly, Australia has seen a variety of improvements in cancer treatment and the development of tailored and targeted therapies which have led to improvements in cancer outcomes [10]. We identified different patterns in cancer incidence for males and females, with the incidence rate for males being relatively stable while the rate for females showed an increase. These were largely driven by lung cancer incidence due to differences in sex-specific smoking behaviours in the population $[3,11]$.

For several cancer types that have an established association with tobacco smoking, including cancers of the lung for males, colorectum, head and neck, stomach, bladder, ovary, oesophagus for females and cervix [12], significant decreases in both cancer incidence and mortality rates have resulted in smaller numbers of new cancer cases and cancer deaths being observed compared to those expected based on rates 20 years ago are likely attributable to Australia's successful tobacco control interventions [3, 4]. However, an increase in the incidence of lung cancer was observed for females, and for lung cancer alone the observed numbers of cases and deaths in 2015 were still substantial, highlighting that continued efforts in tobacco control and the development of effective lung cancer screening and treatments remain significant public health priorities [3]. The first enquiry into the possibility of a national lung cancer screening program is currently progressing in Australia [13].

In the case of colorectal and cervical cancers, organised screening has played a major role in reducing the incidence and mortality rates. Australia's National Cervical Screening Program (NCSP) was implemented in 1991, the reductions in cervical cancer incidence and mortality rates observed in this study can be mainly attributed to the NCSP. However, a previous study reported that the cervical cancer incidence rate appears to have plateaued during the late 2000s following the levelling off of screening participation rates, and a new screening technology and revised protocol were introduced in 2017 [14]. In addition, the national human papillomavirus (HPV) vaccination program was introduced in April 2007 [15], and over time this will be expected to further reduce the incidence rate of cervical cancer [16]. While the National Bowel Cancer Screening Program (NBCSP) has been phased in from 2006, reaching full implementation from 2020 [5], opportunistic screening occurred prior to its launch and continues, so decreases in colorectal cancer incidence and mortality rates are likely to be attributable to both organised and opportunistic screening [17, 18]. However, in contrast to the overall decrease in the incidence and mortality rates for colorectal cancer, a previous study reported increases in the incidence rates for people aged under 50 years [19]. Further research focusing on the increase in the colorectal cancer incidence rate for young people is warranted.

The decreases in the incidence and mortality rates for CUP could potentially be explained by continuous improvements in diagnostic techniques which may have resulted in these cases being correctly classified as a known primary cancer type [20]. Consequently, this could be a potential factor contributing to the increases in the numbers of new diagnoses of other cancer types compared with expected. Reductions in mortality for cancer types commonly thought to be responsible for CUP, including lung cancer, are likely to have also contributed to the smaller than expected number of deaths from CUP [21].

Increasing or relatively stable incidence rates but decreasing mortality rates were observed for some cancer types, including cancers of the prostate, breast, kidney and melanoma. Although prostate cancer and melanoma are often detected early and treated successfully as a result, currently there is no organised population screening for either as the potential harms are considered to outweigh the benefits [22-25]. The decrease in the prostate cancer incidence rate after 2008 [6] is likely to be attributable to clinical guidelines recommending against PSA testing for prostate cancer since 2008 [22, 23]. For breast cancer, despite debate around mammographic 
screening [26, 27], assessments of different methods of screening for breast cancer estimated a reduction in mortality of $21-28 \%$ for women invited to screening $[28,29]$.

As found in the previous study which considered a time period starting a decade earlier than the current study, there were statistically significant increases in both incidence and mortality rates for liver cancer, resulting in significantly larger numbers of liver cancer cases and deaths occurring compared to those expected based on 1995 rates [2]. Multiple factors may be the cause of these increases. The prevalence of hepatitis $B$ and $C$ has remained high in some migrant communities, and among Aboriginal and Torres Strait Islander Australians, and there is a substantial burden of undiagnosed chronic hepatitis infections in high risk groups [30]. Other risk factors such as obesity, diabetes and alcohol consumption may also be a factor [2]. In addition, the misclassification of other cancers which have metastasised to the liver could be a factor [31]. Future research focusing on the increase in incidence and mortality rates for liver cancer is a major priority. This study also found that the mortality rate for cancer of corpus uteri and uterus increased in the recent period from 2010 to 2015, which was likely caused by increases in obesity, diabetes, insufficient physical activity, and decreasing use of estrogen plus progestin menopausal hormone therapy [32, 33].

In the context of the Covid-19 pandemic, health services have been severely disrupted in a number of countries and in Australia cancer services have been disrupted to some extent [34], including a pause of BreastScreen Australia services in April-May 2020. Furthermore, population-level health-seeking may be impacted. It will be important to ensure that these disruptions are minimised and that appropriate recovery strategies are in place; one means by which this is occurring is the establishment of the Covid-19 and Cancer Global Modelling Consortium, which aims to help countries minimise the impact of disruptions [35].

\section{Conclusion}

We found that the cancer mortality rate in Australia in 2015 was about a third lower than the rate in 1995, with a total of almost 107,000 fewer deaths over the 20-year period 1996-2015 than would have been expected had the mortality rate remained at the 1995 level. This is likely to in part reflect the success of past cancer control efforts, especially tobacco control, cancer screening, and improvements in treatment. Despite the improvements observed, an increase in cancer incidence remains a concern, with the number of new cancer diagnoses being greater than that expected based on 1995 rates. Although a proportion of these are likely to be due to increased detection, these findings imply that continuing commitment to further research and the implementation of evidence-based cancer control measures must remain public health priorities.

\section{Limitations}

The main limitation of this analysis is that the estimated changes in incidence and mortality are likely to be sensitive to the reference year selected for comparison. To reduce the impact of this and account for random variations in cancer rates for a single year, the 1995 rates were estimated using a three-year moving average.

\section{Abbreviations \\ APC: Annual percent change; AAPC: Average annual percentage change; AlHW: Australian Institute of Health and Welfare; CDA: Cancer Data in Australia; $\mathrm{Cl}$ : Confidence interval; CUP: Cancers with unknown primary; HPV: Human papilloma virus; ICD: International Classification of Diseases; NBCSP: National Bowel Cancer Screening Program; NCSP: National Cervical Cancer Screening Program; NHL: Non-Hodgkin lymphoma; PSA: Prostate specific antigen.}

\section{Acknowledgements}

We would like to acknowledge the Australian Institute of Health and Welfare who provided the national tabulated data on cancer incidence and mortality. However, they bear no responsibility for the analyses presented within this publication or the interpretation of them. We would like to thank Clare Kahn for editorial assistance.

\section{Authors' contributions}

EF: conceived a similar earlier study. KC: suggested updating the analysis. QL conducted the statistical analysis, interpreted the results and drafted the manuscript. JS contributed to the statistical analysis and methods used. DO'C, $\mathrm{KC}$ and EF contributed to the methods. KC, JS, EF, PG and DO'C contributed to the interpretation of the results and drafting of the manuscript. All authors read and approved the final manuscript.

\section{Funding}

Not applicable.

\section{Availability of data and materials}

The datasets generated and/or analysed for the current study are available from Cancer Data in Australia via: https://www.aihw.gov.au/reports/cancer/ cancer-data-in-australia/contents/summary.

\section{Ethics approval and consent to participate}

Not applicable.

\section{Consent for publication}

Not applicable.

\section{Competing interests}

$\mathrm{KC}$ is CO-PI of an unrelated investigator-initiated trial of cytology and primary HPV screening in Australia ('Compass'), which is conducted and funded by the Victorian Cytology Service (VCS), a government-funded health promotion charity. The VCS has received equipment and a funding contribution for the Compass trial from Roche Molecular Systems and Ventana Inc USA. However, neither KC nor her institution (Cancer Council NSW) receives direct funding from industry for this trial or any other project.

\section{Author details}

${ }^{1}$ Cancer Research Division, Cancer Council NSW, Kings Cross, PO Box 572, Sydney, NSW 1340, Australia. ${ }^{2}$ The University of Sydney School of Public Health, Faculty of Medicine and Health, The University of Sydney, Sydney, NSW, Australia. ${ }^{3}$ School of Medicine and Public Health, University of Newcastle, Newcastle, NSW, Australia. ${ }^{4}$ Prince of Wales Clinical School, University of New South Wales, Sydney, NSW, Australia. 
Received: 22 August 2020 Accepted: 24 November 2020

Published online: 10 December 2020

\section{References}

1. Global Cancer Observatory: cancer today. Lyon, France: International Agency for Research on Cancer. Available from: https://gco.iarc.fr/today. Accessed 3 June 2019

2. Sitas F, Gibberd A, Kahn C, Weber MF, Chiew M, Supramaniam R, Velentzis L, Nickson C, Smith DP, O'Connell D, et al. Cancer incidence and mortality in people aged less than 75 years: changes in Australia over the period 1987-2007. Cancer Epidemiol. 2013:37(6):780-7.

3. Luo Q, Yu XQ, Wade S, Caruana M, Pesola F, Canfell K, O'Connell DL. Lung cancer mortality in Australia: projected outcomes to 2040. Lung Cancer. 2018;125:68-76.

4. Luo Q, Steinberg J, O'Connell DL, Yu XQ, Caruana M, Wade S, Pesola F, Grogan PB, Dessaix A, Freeman B, et al. Lung cancer mortality in Australia in the twenty-first century: How many lives can be saved with effective tobacco control? Lung Cancer. 2019:130:208-15.

5. Australian Institute of Health Welfare. National cancer screening programs participation data. 2020. Cat. No: CAN 114, last updated 29 Jan 2019. Available from: https://www.aihw.gov.au/reports/cancer-screening/ national-cancer-screening-programs-participation/data. Last accessed 30 Jan 2019.

6. Australian Institute of Health and Welfare (AIHW). Cancer data in Australia. Cat. no: CAN 122. 2020. Available from: https://www.aihw.gov.au/reports/ cancer/cancer-data-in-australia/contents/summary. Last updated 2 June 2020. Last accessed 19 Oct 2020

7. Australian Bureau of Statistics. Causes of death, Australia, 2016. Cat. no. 3303.0, ABS, Canberra; 2018

8. National Cancer Institute. Joinpoint Regression Program, Version 4.7.0.0. Released 26 Feb 2019; Statistical Methodology and Applications Branch, Surveillance Research Program, National Cancer Institute. In.

9. StataCorp, . Stata Statistical Software: release 13.1. College Station: StataCorp LP; 2013.

10. Australian Institute of Health and Welfare (AlHW). Australia's health 2014 Australia's health series no. 14. Cat. no. AUS 178. Canberra: AlHW; 2014

11. Adair T, Hoy D, Dettrick Z, Lopez AD. Reconstruction of long-term tobacco consumption trends in Australia and their relationship to lung cancer mortality. Cancer Causes Control CCC. 2011;22(7):1047-53.

12. IARC Working Group on the Evaluation of Carcinogenic Risks to Humans. IARC monographs on the evaluation of carcinogenic risks to humans. Personal habits and indoor combustions vol 100-a review of human carcinogens, part E. Lyon: World Health Organisation; 2012.

13. Cancer Australia Lung Cancer Screening enquiry. https://canceraustralia gov.au/about-us/lung-cancer-screening-enquiry.

14. Smith M, Canfell K. Impact of the Australian National Cervical Screening Program in women of different ages. Med J Aust. 2016;205(8):359-64.

15. Australian Institute of Health Welfare and Australian Government Department of Health and Ageing. Cervical screening in Australia 2008-2009. Cancer series 54. Cat. no. CAN 61. Canberra: AlHW; 2011.

16. Hall MT, Simms KT, Lew J-B, Smith MA, Brotherton JML, Saville M, Frazer $\mathrm{IH}$, Canfell K. The projected timeframe until cervical cancer elimination in Australia: a modelling study. Lancet Public Health. 2019:4(1):e19-27.

17. Stracci F, Zorzi M, Grazzini G. Colorectal cancer screening: tests, strategies, and perspectives. Front Public Health. 2014:2:210

18. Lew JB, St John DJB, Xu XM, Greuter MJE, Caruana M, Cenin DR, He E, Saville M, Grogan P, Coupe VMH, et al. Long-term evaluation of benefits, harms, and cost-effectiveness of the National Bowel Cancer Screening Program in Australia: a modelling study. Lancet Public Health. 2017:2(7):e331-40.

19. Feletto E, Yu XQ, Lew JB, St John DJB, Jenkins MA, Macrae FA, Mahady SE, Canfell K. Trends in colon and rectal cancer incidence in Australia from 1982 to 2014: analysis of data on over 375,000 cases. Cancer Epidemiol Biomarkers Prev. 2019;28(1):83-90.

20. Brustugun OT, Helland A. Rapid reduction in the incidence of cancer of unknown primary. A population-based study. Acta Oncol. 2014;53(1):134-7.

21. Luke C, Koczwara B, Karapetis C, Pittman K, Price T, Kotasek D, Beckmann K, Brown MP, Roder D. Exploring the epidemiological characteristics of cancers of unknown primary site in an Australian population: implications for research and clinical care. Aust NZ J Public Health. 2008;32(4):383-9.

22. Screening for prostate cancer: US Preventive Services Task Force recommendation statement. Ann Intern Med. 2008;149(3):185-91.

23. The Royal Australian College of General Practitioners. Guidelines for preventive activities in general practice (red book). 7th ed. Melbourne: RACGP; 2009.

24. Nufer KL, Raphael AP, Soyer HP. Dermoscopy and overdiagnosis of melanoma in situ. JAMA Dermatol. 2018:154(4):398-9.

25. Pathirana T, Hayen A, Doust J, Glasziou P, Bell K. Lifetime risk of prostate cancer overdiagnosis in Australia: quantifying the risk of overdiagnosis associated with prostate cancer screening in Australia using a novel lifetime risk approach. BMJ Open. 2019;9(3):e022457.

26. Barratt A. Overdiagnosis in mammography screening: a 45 year journey from shadowy idea to acknowledged reality. BMJ. 2015;350:h867.

27. Puliti D, Duffy SW, Miccinesi G, de Koning H, Lynge E, Zappa M, Paci E, Group EW. Overdiagnosis in mammographic screening for breast cancer in Europe: a literature review. J Med Screen. 2012;19(Suppl 1):42-56.

28. BreastScreen Australia. Program evaluation. BreastScreen Australia evaluation final report. Available from: http://cancerscreening.gov.au/internet/ screening/publishing.nsf/Content/programme-evaluation. 2009.

29. IARC Working Group on the Evaluation of Cancer-Preventive Strategies. Breast cancer screening, IARC handbooks of cancer prevention, vol. 15. Lyon: International Agency for Research on Cancer; 2016.

30. The Kirby Institute. Hepatitis B and C in Australia Annual Surveillance Report Supplement 2016. Kensington: The Kirby Institute, UNSW; 2016.

31. Smittenaar CR, Petersen KA, Stewart K, Moitt N. Cancer incidence and mortality projections in the UK until 2035. Br J Cancer. 2016:115(9):1147-55.

32. Henley S, Miller J, Dowling N, Benard V, Richardson L. Uterine cancer incidence and mortality_United States, 1999-2016. MMWR Morb Mortal Wkly Rep. 2018;67(48):1333-8.

33. Australian Institute of Health Welfare. Cancer incidence projections Australia, 2011 to 2020 In., vol. Cancer Series no. 66. Cat. no. CAN 62. Canberra: AlHW; 2012

34. Degeling K, Baxter NN, Emery J, Franchini F, Gibbs P, Mann GB, McArthur $\mathrm{G}$, Solomon BJ, ljzerman MJ. An inverse stage-shift model to estimate the excess mortality and health economic impact of delayed access to cancer services due to the COVID-19 pandemic. medRxiv 2020:2020.2005.2030.20117630.

35. Covid-19 and Cancer Taskforce. https://covidcancertaskforce.org/.

\section{Publisher's Note}

Springer Nature remains neutral with regard to jurisdictional claims in published maps and institutional affiliations. 